\title{
Optimization of the metakaolin geopolymer preparation for maximized ammonium adsorption capacity
}

Tero Luukkonen ${ }^{1}$, Emma-Tuulia Tolonen ${ }^{1,2}$, Hanna Runtti ${ }^{2}$, Kimmo Kemppainen ${ }^{2}$, Paavo Perämäki², Jaakko Rämö², and Ulla Lassi²,3

1. Kajaani University of Applied Sciences, P.O. Box 52, FI-87101 Kajaani Finland

2. University of Oulu, Research Unit of Sustainable Chemistry, P.O. Box 3000, FI-90014 University of Oulu Finland

3. University of Jyvaskyla, Kokkola University Consortium Chydenius, P.O. Box 567, FI67701 Kokkola, Finland

Corresponding author: Tero Luukkonen, Kajaani University of Applied Sciences, P.O. Box 52, FI-87101 Kajaani Finland, teroluuk@gmail.com tero.luukkonen@oulu.fi

Emails of other authors: emma-tuulia.tolonen@oulu.fi; hanna.runtti@oulu.fi; Kimmo.kemppainen@kamk.fi; paavo.peramaki@oulu.fi; Jaakko.ramo@oulu.fi; ulla.lassi@oulu.fi

\begin{abstract}
Geopolymers are functional materials that can be used in various environmental applications such as adsorbents in pollutant removal from wastewaters. Metakaolin geopolymer (MK-GP) has been proven to be especially suitable for ammonium $\left(\mathrm{NH}_{4}{ }^{+}\right)$ removal from wastewater. In this research, the optimal reagent and raw material ratios in the preparation of MK-GP in terms of $\mathrm{NH}_{4}{ }^{+}$adsorption capacity were investigated. The response surface methodology based on the face centered central composite design was used to optimize the levels of three factors: the amounts of hydroxide, silicate, and metakaolin. In addition, the effect of $\mathrm{Na}$ or $\mathrm{K}$ as the charge-balancing cation was studied. Empirical models were fitted to the experimental data using multiple linear regression. The significance of the models was confirmed by means of analysis of variance. Optimal $\mathrm{NH}_{4}{ }^{+}$removal efficiency was achieved when the amount of hydroxide and silicate were maximized, the amount of metakaolin was minimized, and Na-based reagents were used. These trends are most likely a result of optimized conversion of metakaolin into MK-GP.
\end{abstract}


Keywords: adsorption; ammonium removal; geopolymer; statistical experiment design; wastewater treatment

\section{Introduction}

Geopolymers are defined as materials formed by a reaction between alkali activator (concentrated aqueous solution of e.g. alkali hydroxide and silicate) and solid aluminosilicate precursors (such as metakaolin or fly ash) [1, 2]. However, also materials obtained from a reaction between alumino-silicate precursors and phosphoric acid are sometimes referred to as acid-activated geopolymers [3]. Geopolymers have recently gained interest in several water and wastewater treatment applications such as adsorbents [4-11], membrane material [12], photocatalysts [13, 14], functional construction materials $[15,16]$, or substrate for biofilm reactors [17]. In many of the aforementioned applications, the use of geopolymers is based on their ion exchange capacity and porous structure.

One interesting use for metakaolin-based geopolymer (MK-GP) is in ammonium $\left(\mathrm{NH}_{4}{ }^{+}\right)$ removal from wastewater [18]. $\mathrm{NH}_{4}{ }^{+}$is the most significant nitrogen species contributing to the eutrophication of water bodies when nitrogen is the nutrient in shortest supply. The main advantage of MK-GP over the conventional biological nitrificationdenitrification process is the lower dependency on temperature since it works as a cation exchanger [18]. Furthermore, MK-GP has been shown to be regenerable, more effective than typical natural zeolites, and simpler to produce than synthetic zeolites which require higher synthesis temperature $[18,19]$.

Although there is already an appreciable amount of papers published on the adsorption properties of geopolymers, only few studies have aimed to systematically determine the impact of preparation conditions on adsorption efficiency. Wang et al. [20] and Li et al. [21] showed that the increased curing temperature and the sodium to fly ash weight ratio up to a certain point improved the $\mathrm{Cu}^{2+}$ removal efficiency when preparing fly ashbased geopolymers by the fusion method. It is known that the elevated temperature promotes the formation of crystalline zeolitic phases [22] and specific surface area of geopolymers correlates linearly with a geopolymer sample zeolite content [23]. Furthermore, the amounts of $\mathrm{SiO}_{2}, \mathrm{Al}_{2} \mathrm{O}_{3}, \mathrm{H}_{2} \mathrm{O}, \mathrm{OH}^{-}$, and cations in the reaction mixture affect the structure of geopolymer [24]. However, the relationship between geopolymer preparation parameters and adsorption efficiency is not well understood.

In order to obtain a maximal $\mathrm{NH}_{4}{ }^{+}$adsorption capacity and to understand the impact of preparation conditions and formulation, the synthesis of MK-GP needs to be optimized. Statistical experimental design is a reliable approach for studying the effects of experimental variables and to optimize their levels in many branches of science [25]. A face centered central composite design (CCF) was used in this study to optimize the preparation conditions of MK-GPs. CCFs provide information on the effects of experimental variables with a minimum number of experiments. A response surface model can be fitted to the experimental data. After validation, the model will show how changes in variable levels affect a response of interest and can be used to find the levels of variables that will optimize the response. A CCF design requires three levels for each factor. 
In this research, the influences of the amounts of hydroxide, silicate, and metakaolin used for the preparation of MK-GPs on $\mathrm{NH}_{4}{ }^{+}$adsorption were investigated. Furthermore, the experiments were conducted with $\mathrm{Na}$ and $\mathrm{K}$ hydroxide and silicate to observe the effect of the charge-balancing cation. The experiments for the preparation of MK-GPs were carried out according to CCF, the geopolymers were characterized for their physico-chemical structure, and tested for $\mathrm{NH}_{4}{ }^{+}$removal using model solutions. The experimental design was prepared and the data was analyzed using the MODDE software (www.umetrics.com).

\section{Materials and methods}

\subsection{Experimental design}

The effects of three factors on the response was investigated and optimized using a CCF design. Factors represent the corresponding amounts of hydroxide, silicate and metakaolin used in the preparation of geopolymer specimen. The levels of the factors (Table 1) were selected according to our previous research [18]. 17 experiments as duplicates (total 34 experiments) were conducted in a random order for both $\mathrm{Na}$ and $\mathrm{K}$ based MK-GPs (Table 2). Statistical analysis of the results gained from the batch adsorption experiments were performed with the MODDE 9.1 software. A quadratic model was fitted to the data using multiple linear regression (MLR) and the significance of the model was tested by means of analysis of variance (ANOVA) with a 95\% confidence level.

Table 1. The levels of experimental factors in the CCF design.

\begin{tabular}{lllllll}
\hline Factor & \multicolumn{3}{l}{ Na-based MK-GPs } & \multicolumn{3}{l}{ K-based MK-GPs } \\
\cline { 2 - 7 } & $\begin{array}{l}\text { Low }(- \\
\text { 1) }\end{array}$ & $\begin{array}{l}\text { Intermediate } \\
(0)\end{array}$ & High (+1) & $\begin{array}{l}\text { Low }(- \\
1)\end{array}$ & $\begin{array}{l}\text { Intermediate } \\
(0)\end{array}$ & $\begin{array}{l}\text { High } \\
(+1)\end{array}$ \\
\hline A: Hydroxide & 6.24 & 7.455 & 8.67 & 8.29 & 9.81 & 11.33 \\
[g] & & & & & & \\
B: Silicate [g] & 7.95 & 8.835 & 9.72 & 8.07 & 8.965 & 9.86 \\
C: Metakaolin & 50 & 65 & 80 & 50 & 65 & 80 \\
[g] & & & & & & \\
\hline
\end{tabular}


Table 2. Design matrix for the three-level CCF design.

\begin{tabular}{llllll}
\hline \multirow{2}{*}{ Exp No } & \multirow{2}{*}{ Exp Name } & \multirow{2}{*}{ Run Order } & \multicolumn{3}{l}{ Levels of factors } \\
\cline { 4 - 6 } & & & A: Hydroxide & B: Silicate & C: Metakaolin \\
\hline 1 & MK-M-N1 & 7 & -1 & -1 & -1 \\
2 & MK-M-N2 & 5 & +1 & -1 & -1 \\
3 & MK-M-N3 & 16 & -1 & +1 & -1 \\
4 & MK-M-N4 & 14 & +1 & +1 & -1 \\
5 & MK-M-N5 & 4 & -1 & -1 & +1 \\
6 & MK-M-N6 & 17 & +1 & -1 & +1 \\
7 & MK-M-N7 & 11 & -1 & +1 & +1 \\
8 & MK-M-N8 & 8 & +1 & +1 & +1 \\
9 & MK-M-N9 & 15 & -1 & 0 & 0 \\
10 & MK-M-N10 & 6 & +1 & 0 & 0 \\
11 & MK-M-N11 & 2 & 0 & -1 & 0 \\
12 & MK-M-N12 & 9 & 0 & +1 & 0 \\
13 & MK-M-N13 & 10 & 0 & 0 & -1 \\
14 & MK-M-N14 & 13 & 0 & 0 & +1 \\
15 & MK-M-N15 & 12 & 0 & 0 & 0 \\
16 & MK-M-N16 & 3 & 0 & 0 & 0 \\
17 & MK-M-N17 & 1 & 0 & 0 & 0 \\
\hline
\end{tabular}

$\mathrm{a}=\mathrm{M}$ is $\mathrm{Na}$ or $\mathrm{K},{ }^{\mathrm{b}}=$ coded levels, for real levels, see Table 1.

\subsection{Preparation of metakaolin geopolymers}

The reagents used for the preparation of GPs were $\mathrm{NaOH}$ (VWR Chemicals, > 97\%), $\mathrm{Na}$ silicate (VWR Chemicals, $\mathrm{SiO}_{2} 26.8 \%, \mathrm{Na}_{2} \mathrm{O} 8.2 \%$ ), $\mathrm{KOH}$ (Merck, $\geq 85 \%$ ), and $\mathrm{K}$ silicate (obtained from a Finnish supplier, Sateenkaari Perinnetaito Ltd, $\mathrm{SiO}_{2} 24.5 \%, \mathrm{~K}_{2} \mathrm{O}$ 11\%). Powdered metakaolin was obtained from Aquaminerals Finland Ltd.

The geopolymers were prepared as follows: 8,10 , or $12 \mathrm{M} \mathrm{NaOH}$ or $\mathrm{KOH}$ solution was mixed with $\mathrm{Na}$ or $\mathrm{K}$ silicate $(0.8: 1,1: 1$, or $1.2: 1 \mathrm{w} / \mathrm{w})$ in order to obtain the levels shown in Table 1. The alkaline solution was allowed to stand for $24 \mathrm{~h}$ before use, in order to allow the silicate solution to depolymerize. Metakaolin was then mixed with the alkaline solution in the ratios $(\mathrm{w} / \mathrm{w})$ of $1: 1,1.3: 1$, or $1.6: 1$ in order to obtain the amounts according to the CCF design (Table 1 ). The paste was then mixed for five minutes and allowed to consolidate at room temperature for three days. The geopolymer specimens were then crushed and sieved to the particle size of 63-125 $\mu \mathrm{m}$. Before use, the materials were washed carefully with distilled water, dried at $+105^{\circ} \mathrm{C}$ and stored in a desiccator.

\subsection{Characterization of metakaolin geopolymers}

The crystalline phases in MK-GPs were characterized with an X-ray diffractometer (PanAnalytical Xpert Pro) using Co K $\alpha$ radiation generated at $40 \mathrm{kV}$ and $40 \mathrm{~mA}$. XRD patterns were collected in the range of $5-80^{\circ} 2 \theta$ using a scan time of $1.25 \mathrm{~s}$ per $0.02^{\circ}$. Samples were prepared by dispensing a finely ground specimen with ethanol onto a glass plate and allowing the ethanol to vaporize before measurement. Diffractograms 
were interpreted using the Highscore software (version 3.0) and the Chrystallography Open Database, the 2013 version.

The elemental composition of samples was determined semiquantitatively with an Xray fluorescence (XRF) spectrometer (PanAnalytical Minipal 4) and the Omnian software. Samples were ground to a fine powder and compressed to tablets before analysis. Loss on ignition (LOI) value was determined using a thermogravimeter (Linseis STA PT1000) from dried powdered samples by heating them to $950^{\circ} \mathrm{C}$.

Specific surface area and pore volumes of samples were determined using $\mathrm{N}_{2}$ gas adsorption-desorption isotherms at the temperature of liquid nitrogen $\left(-196^{\circ} \mathrm{C}\right)$ by using a Micrometrics ASAP 2020 instrument. Specific surface area was calculated based on the Brunauer-Emmett-Teller (BET) isotherm. Pore size distributions were calculated from desorption data using the Barrett-Joyner-Halenda (BJH) method.

\subsection{Determination of ammonium removal efficiency}

$\mathrm{NH}_{4}{ }^{+}$adsorption experiments for each prepared geopolymer specimen were conducted as batch experiments at room temperature $\left(22^{\circ} \mathrm{C}\right)$. A model solution $\left(\mathrm{NH}_{4}{ }^{+}=140 \mathrm{mg} / \mathrm{l}\right)$ whose $\mathrm{pH}$ was adjusted to 6.0 before experiments was prepared by dissolving $\mathrm{NH}_{4} \mathrm{Cl}$ (Merck) into ultrapure water. A constant dose of $5 \mathrm{~g} / \mathrm{L}$ of MK-GP and a $24 \mathrm{~h}$ mixing time (in which equilibrium was reached) were used in every adsorption experiment. After mixing, MK-GP powder was separated by centrifuging and the supernatant was analyzed for $\mathrm{NH}_{4}^{+}$concentration using a flow injection analyzer (Foss-Tecator Fiastar 5000).

\section{Results and discussion}

\subsection{Characterization of the prepared metakaolin geopolymers}

The prepared geopolymer specimens used in experimental runs were labelled as MKNa-N1 to MK-Na-N17 and MK-K-N1 to MK-K-N17 as shown in Table 2.

According to the XRD results (Fig. 1), there are no clear differences between the samples: all samples contain quartz $\left(\mathrm{SiO}_{2}\right)$ and muscovite $\left(\mathrm{KAl}_{2}\left(\mathrm{AlSi}_{3} \mathrm{O}_{10}\right)(\mathrm{OH})_{2}\right)$. Additionally, all samples have an amorphous halo between approx. $25-35^{\circ} 2 \theta$ which is associated with the unordered geopolymer structure and possibly unreacted metakaolin. Although there are no zeolite peaks present, it has been argued that the formation of nanocrystalline zeolites (crystals too small to cause X-ray diffraction) could take place during geopolymer formation [26]. 

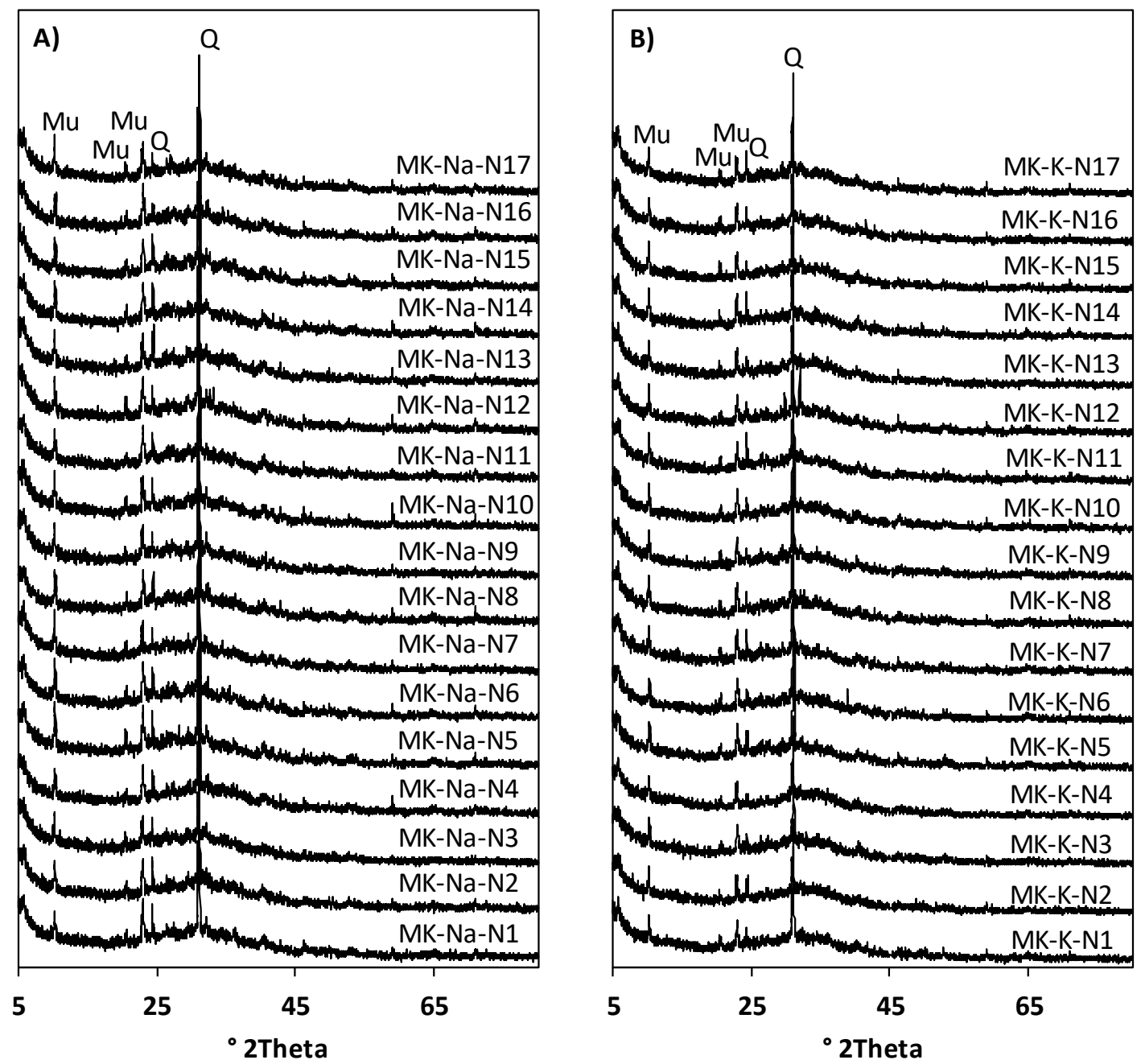

Fig. 1. X-ray diffraction patterns of A) Na-based and B) K-based MK-GPs. Mu = muscovite $\left(\mathrm{KAl}_{2}\left(\mathrm{AlSi}_{3} \mathrm{O}_{10}\right)(\mathrm{OH})_{2}, \mathrm{Q}=\right.$ quartz $\left(\mathrm{SiO}_{2}\right)$.

The elemental composition of the geopolymer specimens are shown in Table 3 . The geopolymer structure is thought to consist of an irregular, i.e. amorphous, aluminosilicate network [27]. $\mathrm{K}_{2} \mathrm{O}$ in Na-based series originates from muscovite $\left(\mathrm{KAl}_{2}\left(\mathrm{AlSi}_{3} \mathrm{O}_{10}\right)(\mathrm{OH})_{2}\right)$ which was present as an impurity in metakaolin. Other minor constituents $\left(\mathrm{SO}_{3}, \mathrm{Ti}\right.$, and $\left.\mathrm{Fe}_{2} \mathrm{O}_{3}\right)$ are related to amorphous impurity phases as there were no other phases than quartz and muscovite detected by XRD. The $\mathrm{SiO}_{2} / \mathrm{Al}_{2} \mathrm{O}_{3}$ molar ratio calculated from the results gives an approximation about the charge density of the aluminosilicate framework structure: the lower value is related to a more negative charge as the Al tetrahedron, $\left[\mathrm{AlO}_{4}\right]^{-}$, are negatively charged [28]. However, also quartz and muscovite dedected by XRD affect the $\mathrm{SiO}_{2} / \mathrm{Al}_{2} \mathrm{O}_{3}$ ratio in addition to the actual geopolymer framework structure and thus the $\mathrm{SiO}_{2} / \mathrm{Al}_{2} \mathrm{O}_{3}$ ratio in this case gives only a rough estimation about the charge density. There seems to be no clear linear correlation between the $\mathrm{SiO}_{2} / \mathrm{Al}_{2} \mathrm{O}_{3}$ molar ratio and $\mathrm{NH}_{4}{ }^{+}$removal capacity (shown in Tables 5 and 6). The $\mathrm{Na}_{2} \mathrm{O}$ and $\mathrm{K}_{2} \mathrm{O}$ concentrations indicate the amounts of exchangeable cations in geopolymers and in fact there is a rather clear linear correlation between $\mathrm{Na}_{2} \mathrm{O}$ or $\mathrm{K}_{2} \mathrm{O}$ 
and the $\mathrm{NH}_{4}{ }^{+}$adsorption capacity (Fig. 2). According to the literature, a higher $\mathrm{Na} / \mathrm{Al}$ (or $\mathrm{K} / \mathrm{Al}$ ) ratio increases the formation of zeolitic phases similarly to increased water content $[26,29]$. There were, however, no observable zeolite peaks present in the XRD results. The loss on ignition (LOI) value indicates the amount of water incorporated in the geopolymer structure. In addition, the calculated molar ratios of $\mathrm{M}_{2} \mathrm{O} / \mathrm{SiO}_{2}$ and $\mathrm{H}_{2} \mathrm{O} / \mathrm{M}_{2} \mathrm{O}(\mathrm{M}=\mathrm{Na}$ or $\mathrm{K})$ are provided in Table 3. According to a review by Khale and Chaudhary [30], geopolymers have typically $\mathrm{SiO}_{2} / \mathrm{Al}_{2} \mathrm{O}_{3}, \mathrm{M}_{2} \mathrm{O} / \mathrm{SiO}_{2}$, and $\mathrm{H}_{2} \mathrm{O} / \mathrm{M}_{2} \mathrm{O}$ molar ratios within 3.3-4.5, $0.2-0.48$ and $10-25$, respectfully. Some of the samples prepared in this study have values below or above the stated ranges. However, those typical ratios reported in the literature are related to optimizing mechanical properties such as compressive strength which are not a priority in the present study. Also, if waste materials are used in geopolymer preparation, the typical ranges are not necessarily applicable [30].

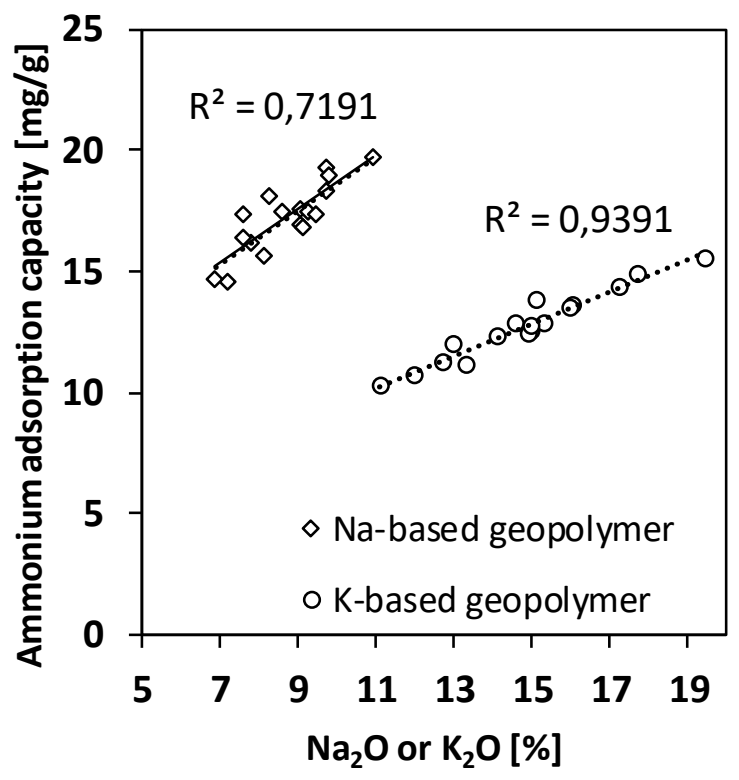

Fig. 2. The linear correlation between $\mathrm{Na}_{2} \mathrm{O}$ or $\mathrm{K}_{2} \mathrm{O}$ and the observed $\mathrm{NH}_{4}{ }^{+}$adsorption capacity. 
Table 3. Elemental composition, loss on ignition (LOI), and calculated molar ratios of $\mathrm{Na}$ and K-based MK-GPs.

\begin{tabular}{|c|c|c|c|c|c|c|c|c|c|c|c|}
\hline \multirow{2}{*}{ Sample } & \multicolumn{7}{|c|}{ Elemental composition $[\%, \mathrm{w} / \mathrm{w}]$} & \multirow{2}{*}{$\begin{array}{l}\text { LOI }[\%, \\
w / w]\end{array}$} & \multicolumn{3}{|c|}{ Calculated molar ratios } \\
\hline & $\mathrm{Na}_{2} \mathrm{O}$ & $\mathrm{Al}_{2} \mathrm{O}_{3}$ & $\mathrm{SiO}_{2}$ & $\mathrm{SO}_{3}$ & $\mathrm{~K}_{2} \mathrm{O}$ & $\mathrm{Ti}$ & $\mathrm{Fe}_{2} \mathrm{O}_{3}$ & & $\mathrm{SiO}_{2} / \mathrm{Al}_{2} \mathrm{O}_{3}$ a & $\mathrm{M}_{2} \mathrm{O} / \mathrm{SiO}_{2} \mathrm{~b}$ & $\mathrm{H}_{2} \mathrm{O} / \mathrm{M}_{2} \mathrm{O}^{\mathrm{c}}$ \\
\hline MK-Na-N1 & 8.26 & 23.84 & 42.30 & 0.13 & 1.99 & 0.06 & 1.52 & 10.51 & 3.01 & 0.19 & 25.93 \\
\hline MK-Na-N2 & 9.70 & 23.72 & 40.96 & 0.11 & 1.95 & 0.05 & 1.45 & 12.55 & 2.93 & 0.23 & 20.71 \\
\hline MK-Na-N3 & 9.72 & 25.27 & 41.65 & 0.08 & 2.03 & 0.05 & 1.48 & 12.78 & 2.80 & 0.23 & 27.31 \\
\hline MK-Na-N4 & 10.95 & 23.98 & 39.63 & 0.07 & 1.97 & 0.05 & 1.48 & 10.52 & 2.80 & 0.27 & 22.42 \\
\hline MK-Na-N5 & 7.18 & 31.14 & 48.49 & 0.08 & 2.27 & 0.06 & 1.64 & 8.01 & 2.64 & 0.14 & 29.83 \\
\hline MK-Na-N6 & 7.76 & 28.80 & 45.06 & 0.07 & 2.23 & 0.06 & 1.61 & 9.02 & 2.66 & 0.17 & 25.89 \\
\hline MK-Na-N7 & 6.88 & 27.55 & 42.42 & 0.07 & 2.29 & 0.06 & 1.71 & 8.10 & 2.61 & 0.16 & 38.59 \\
\hline MK-Na-N8 & 9.07 & 29.78 & 45.29 & 0.09 & 2.23 & 0.06 & 1.62 & 9.15 & 2.58 & 0.19 & 27.07 \\
\hline MK-Na-N9 & 7.59 & 26.22 & 42.21 & 0.07 & 2.17 & 0.06 & 1.60 & 10.10 & 2.73 & 0.17 & 31.60 \\
\hline MK-Na-N10 & 8.62 & 24.99 & 40.37 & 0.11 & 2.04 & 0.07 & 1.54 & 11.79 & 2.74 & 0.21 & 25.89 \\
\hline MK-Na-N11 & 7.58 & 25.01 & 41.62 & 0.08 & 2.08 & 0.06 & 1.56 & 9.61 & 2.82 & 0.18 & 27.33 \\
\hline MK-Na-N12 & 9.05 & 26.59 & 41.89 & 0.07 & 2.15 & 0.06 & 1.60 & 8.15 & 2.67 & 0.21 & 28.18 \\
\hline MK-Na-N13 & 9.82 & 24.56 & 41.36 & 0.07 & 2.00 & 0.05 & 1.49 & 11.75 & 2.86 & 0.23 & 23.53 \\
\hline MK-Na-N14 & 8.15 & 30.45 & 46.59 & 0.08 & 2.27 & 0.06 & 1.63 & 6.90 & 2.60 & 0.17 & 28.35 \\
\hline MK-Na-N15 & 9.12 & 28.20 & 44.68 & 0.07 & 2.15 & 0.06 & 1.57 & 7.70 & 2.69 & 0.20 & 25.34 \\
\hline MK-Na-N16 & 9.27 & 28.62 & 45.43 & 0.07 & 2.15 & 0.06 & 1.58 & 7.39 & 2.69 & 0.20 & 24.93 \\
\hline MK-Na-N17 & 9.48 & 28.73 & 45.59 & 0.07 & 2.16 & 0.06 & 1.57 & 8.52 & 2.69 & 0.20 & 24.38 \\
\hline MK-K-N1 & 0.00 & 23.26 & 39.30 & 0.07 & 15.11 & 0.05 & 1.54 & 8.83 & 2.87 & 0.25 & 20.31 \\
\hline MK-K-N2 & 0.00 & 23.56 & 39.23 & 0.06 & 17.70 & 0.06 & 1.50 & 9.44 & 2.83 & 0.29 & 15.92 \\
\hline MK-K-N3 & 0.00 & 24.70 & 39.76 & 0.06 & 16.08 & 0.06 & 1.55 & 8.08 & 2.73 & 0.26 & 23.40 \\
\hline MK-K-N4 & 0.00 & 24.90 & 39.85 & 0.06 & 19.47 & 0.05 & 1.50 & 11.43 & 2.72 & 0.31 & 17.39 \\
\hline MK-K-N5 & 0.00 & 26.72 & 41.29 & 0.07 & 11.12 & 0.06 & 1.67 & 6.28 & 2.62 & 0.17 & 27.60 \\
\hline MK-K-N6 & 0.00 & 25.16 & 38.96 & 0.06 & 13.02 & 0.06 & 1.64 & 6.35 & 2.63 & 0.21 & 21.65 \\
\hline MK-K-N7 & 0.00 & 30.12 & 45.26 & 0.07 & 11.96 & 0.06 & 1.66 & 6.69 & 2.55 & 0.17 & 31.46 \\
\hline MK-K-N8 & 0.00 & 29.00 & 43.57 & 0.07 & 14.56 & 0.06 & 1.63 & 6.32 & 2.55 & 0.21 & 23.26 \\
\hline MK-K-N9 & 0.00 & 29.15 & 45.21 & 0.07 & 13.34 & 0.06 & 1.59 & 6.73 & 2.63 & 0.19 & 25.61 \\
\hline MK-K-N10 & 0.00 & 28.07 & 43.75 & 0.06 & 15.96 & 0.06 & 1.56 & 9.54 & 2.65 & 0.23 & 19.44 \\
\hline MK-K-N11 & 0.00 & 28.13 & 44.53 & 0.06 & 14.14 & 0.06 & 1.57 & 8.85 & 2.69 & 0.20 & 20.78 \\
\hline MK-K-N12 & 0.00 & 28.21 & 43.37 & 0.07 & 15.31 & 0.06 & 1.58 & 8.97 & 2.61 & 0.23 & 23.29 \\
\hline MK-K-N13 & 0.00 & 25.05 & 40.67 & 0.06 & 17.28 & 0.06 & 1.49 & 10.06 & 2.76 & 0.27 & 18.82 \\
\hline MK-K-N14 & 0.16 & 29.86 & 45.09 & 0.07 & 12.75 & 0.06 & 1.62 & 6.51 & 2.56 & 0.18 & 25.50 \\
\hline MK-K-N15 & 0.00 & 28.39 & 44.10 & 0.07 & 14.98 & 0.06 & 1.57 & 8.48 & 2.64 & 0.22 & 21.70 \\
\hline MK-K-N16 & 0.00 & 27.68 & 43.01 & 0.06 & 14.91 & 0.06 & 1.58 & 8.35 & 2.64 & 0.22 & 21.81 \\
\hline MK-K-N17 & 0.00 & 27.61 & 42.88 & 0.06 & 14.96 & 0.06 & 1.58 & 11.38 & 2.64 & 0.22 & 21.73 \\
\hline
\end{tabular}

${ }^{a}=$ calculated from the XRF data, ${ }^{b}=$ calculated from the XRF data, $c=$ calculated form the XRF data and added water from hydroxide and silicate solutions. $\mathrm{M}=\mathrm{Na}$ or $\mathrm{K}$. The best-performing $\mathrm{NH}_{4}{ }^{+}$adsorbents (MK-Na-N4 and MK-K-N4) are bolded.

The specific surface area and pore volume distribution between micro $\left(\mathrm{d}_{0}<2 \mathrm{~nm}\right)$, meso $\left(2 \mathrm{~nm}<\mathrm{d}_{0}<50 \mathrm{~nm}\right)$ and macro $\left(\mathrm{d}_{0}>50 \mathrm{~nm}\right)$ pores are shown in Table 4. The Na-based geopolymers have clearly lower specific surface area than K-based geopolymers. However, there is no linear correlation between the specific surface area and $\mathrm{NH}_{4}{ }^{+}$ 
adsorption capacity in the results of the GPs. On the other hand, the average pore size is larger in the Na-based series. The reported aqueous ionic radiis of $\mathrm{Na}, \mathrm{K}$, and $\mathrm{NH}_{4}{ }^{+}$are $0.235,0.279$, and $0.260 \mathrm{~nm}$, respectively, which demonstrates that only micropores have a role in the adsorption phenomena [31]. As a trend, the specific surface area increases as the $\mathrm{Si} / \mathrm{Al}$ ratio is increased: this is more evident in the K-based series. 
Table 4. Specific surface areas and pore volume distributions of Na and K-based MK-GPs.

\begin{tabular}{|c|c|c|c|c|c|c|c|c|c|}
\hline Sample & $\begin{array}{l}\text { BET } \\
\text { area } \\
{\left[\mathrm{m}^{2} / \mathrm{g}\right.} \\
]\end{array}$ & $\begin{array}{l}\text { Av. } \\
\text { pore } \\
\text { size } \\
{[\mathrm{nm}]}\end{array}$ & $\begin{array}{l}\text { Micro } \\
\text { pores } \\
{\left[\mathrm{cm}^{3} / \mathrm{g}\right]}\end{array}$ & $\begin{array}{l}\text { Meso } \\
\text { pores } \\
{\left[\mathrm{cm}^{3} / \mathrm{g}\right]}\end{array}$ & $\begin{array}{l}\text { Macro } \\
\text { pores } \\
{\left[\mathrm{cm}^{3} / \mathrm{g}\right]}\end{array}$ & $\begin{array}{l}\text { Total } \\
\text { volume } \\
{\left[\mathrm{cm}^{3} / \mathrm{g}\right]}\end{array}$ & $\begin{array}{l}\text { Micro } \\
\text { pores } \\
{[\%]}\end{array}$ & $\begin{array}{l}\text { Meso } \\
\text { pores } \\
{[\%]}\end{array}$ & $\begin{array}{l}\text { Macro } \\
\text { pores } \\
{[\%]}\end{array}$ \\
\hline MK-Na-N1 & 37.8 & 26.8 & 0.002 & 0.116 & 0.134 & 0.252 & 0.61 & 46.12 & 53.27 \\
\hline MK-Na-N2 & 35.8 & 20.8 & 0.001 & 0.145 & 0.039 & 0.185 & 0.53 & 78.46 & 21.00 \\
\hline MK-Na-N3 & 23.7 & 26.0 & 0.001 & 0.080 & 0.072 & 0.153 & 0.56 & 52.38 & 47.05 \\
\hline MK-Na-N4 & 19.3 & 30.3 & 0.000 & 0.106 & 0.040 & 0.147 & 0.26 & 72.28 & 27.46 \\
\hline MK-Na-N5 & 31.6 & 24.6 & 0.001 & 0.106 & 0.088 & 0.195 & 0.50 & 54.44 & 45.06 \\
\hline MK-Na-N6 & 27.2 & 22.2 & 0.001 & 0.107 & 0.042 & 0.150 & 0.55 & 71.21 & 28.24 \\
\hline MK-Na-N7 & 19.1 & 31.2 & 0.001 & 0.064 & 0.083 & 0.148 & 0.38 & 43.45 & 56.16 \\
\hline MK-Na-N8 & 19.8 & 27.4 & 0.001 & 0.060 & 0.074 & 0.135 & 0.66 & 44.60 & 54.74 \\
\hline MK-Na-N9 & 28.3 & 26.2 & 0.001 & 0.095 & 0.087 & 0.184 & 0.66 & 51.92 & 47.42 \\
\hline MK-Na-N10 & 26.9 & 27.8 & 0.001 & 0.086 & 0.100 & 0.187 & 0.60 & 45.84 & 53.55 \\
\hline MK-Na-N11 & 32.5 & 28.2 & 0.001 & 0.115 & 0.112 & 0.227 & 0.40 & 50.37 & 49.23 \\
\hline MK-Na-N12 & 23.3 & 27.2 & 0.001 & 0.076 & 0.081 & 0.157 & 0.60 & 48.01 & 51.39 \\
\hline MK-Na-N13 & 28.5 & 29.9 & 0.001 & 0.089 & 0.121 & 0.212 & 0.46 & 42.10 & 57.44 \\
\hline MK-Na-N14 & 25.3 & 26.0 & 0.001 & 0.082 & 0.081 & 0.163 & 0.62 & 49.96 & 49.42 \\
\hline MK-Na-N15 & 25.0 & 32.1 & 0.001 & 0.079 & 0.119 & 0.199 & 0.39 & 39.73 & 59.88 \\
\hline MK-Na-N16 & 25.6 & 26.9 & 0.001 & 0.085 & 0.084 & 0.171 & 0.70 & 49.83 & 49.48 \\
\hline MK-Na-N17 & 23.4 & 28.9 & 0.001 & 0.078 & 0.089 & 0.168 & 0.58 & 46.67 & 52.75 \\
\hline MK-K-N1 & 94.0 & 15.1 & 0.002 & 0.309 & 0.044 & 0.355 & 0.40 & 87.20 & 12.40 \\
\hline MK-K-N2 & 110.0 & 12.5 & 0.002 & 0.300 & 0.040 & 0.342 & 0.50 & 87.90 & 11.60 \\
\hline MK-K-N3 & 88.0 & 17.9 & 0.002 & 0.364 & 0.030 & 0.396 & 0.40 & 92.00 & 7.60 \\
\hline MK-K-N4 & 59.0 & 18.5 & 0.001 & 0.232 & 0.038 & 0.270 & 0.30 & 85.70 & 14.00 \\
\hline MK-K-N5 & 80.0 & 13.9 & 0.002 & 0.245 & 0.030 & 0.277 & 0.60 & 88.70 & 10.70 \\
\hline MK-K-N6 & 72.0 & 13.4 & 0.002 & 0.201 & 0.037 & 0.240 & 0.80 & 83.80 & 15.40 \\
\hline MK-K-N7 & 64.0 & 17.0 & 0.001 & 0.247 & 0.021 & 0.268 & 0.40 & 91.90 & 7.70 \\
\hline MK-K-N8 & 52.0 & 17.8 & 0.001 & 0.193 & 0.037 & 0.231 & 0.50 & 83.60 & 15.80 \\
\hline MK-K-N9 & 81.0 & 15.8 & 0.002 & 0.290 & 0.026 & 0.318 & 0.50 & 91.20 & 8.30 \\
\hline MK-K-N10 & 68.0 & 14.7 & 0.002 & 0.204 & 0.044 & 0.249 & 0.60 & 81.80 & 17.60 \\
\hline MK-K-N11 & 84.0 & 13.8 & 0.002 & 0.254 & 0.033 & 0.289 & 0.60 & 88.00 & 11.30 \\
\hline MK-K-N12 & 60.0 & 18.0 & 0.001 & 0.240 & 0.027 & 0.268 & 0.40 & 89.60 & 10.00 \\
\hline MK-K-N13 & 82.0 & 15.2 & 0.002 & 0.263 & 0.048 & 0.312 & 0.50 & 84.10 & 15.50 \\
\hline MK-K-N14 & 64.0 & 16.0 & 0.001 & 0.221 & 0.034 & 0.257 & 0.40 & 86.20 & 13.40 \\
\hline MK-K-N15 & 73.0 & 15.5 & 0.002 & 0.224 & 0.056 & 0.282 & 0.60 & 79.70 & 19.80 \\
\hline MK-K-N16 & 79.0 & 14.8 & 0.002 & 0.233 & 0.057 & 0.292 & 0.80 & 79.70 & 19.50 \\
\hline MK-K-N17 & 60.0 & 18.8 & 0.000 & 0.225 & 0.058 & 0.282 & 0.00 & 79.60 & 20.40 \\
\hline
\end{tabular}

The best-performing $\mathrm{NH}_{4}{ }^{+}$adsorbents (MK-Na-N4 and MK-K-N4) are in bold. Micropores: $\mathrm{d}_{0}<$ $2 \mathrm{~nm}$, mesopores $2<\mathrm{d}_{0}<50 \mathrm{~nm}$, macropores $\mathrm{d}_{0}>50 \mathrm{~nm}$ 


\subsection{Optimization of geopolymer preparation conditions for ammonium removal}

The experimental results for $\mathrm{NH}_{4}{ }^{+}$adsorption capacities obtained with CCF designs for $\mathrm{Na}$ and K-based MK-GPs are shown in Tables 5 and 6 . The best $\mathrm{NH}_{4}{ }^{+}$removal was achieved with samples MK-Na-N4 and MK-K-N4. The Na-based geopolymers had 27$48 \%$ higher $\mathrm{NH}_{4}{ }^{+}$removal capacity than the K-based geopolymers. It should be noted that the reported capacities do not represent the theoretical maximum adsorption capacity but the capacity obtained within the experimental conditions ( $5 \mathrm{~g} / \mathrm{L}$ adsorbent dose, $140 \mathrm{mg} / \mathrm{L}$ initial $\mathrm{NH}_{4}{ }^{+}$concentration, and $24 \mathrm{~h}$ mixing time). The maximum adsorption capacity of the best material (MK-Na-N4) in model solutions was determined to be $31.79 \mathrm{mg} / \mathrm{g}$ in another study [32]. This is above the typical maximum $\mathrm{NH}_{4}{ }^{+}$ adsorption capacities of natural zeolites and is exceeded only by few synthetic zeolites reported in the literature [18]. 
Table 5. The results of the $\mathrm{NH}_{4}{ }^{+}$removal tests with Na-based MK-GPs.

\begin{tabular}{|c|c|c|c|c|c|}
\hline \multirow[b]{2}{*}{$\begin{array}{l}\text { Experimental } \\
\text { run (sample) }\end{array}$} & \multicolumn{3}{|c|}{ Factor/level } & \multirow{2}{*}{$\begin{array}{l}\text { Observed } \\
\mathrm{NH}_{4}{ }^{+} \\
\text {adsorption } \\
\text { capacity } \\
\text { [mg/g] } \\
\text { experiment } 1\end{array}$} & \multirow{2}{*}{$\begin{array}{l}\text { Observed } \\
\mathrm{NH}_{4}^{+} \\
\text {adsorption } \\
\text { capacity } \\
\text { [mg/g] } \\
\text { experiment } 2\end{array}$} \\
\hline & $\begin{array}{l}\text { A: } \mathrm{NaOH} \\
{[\mathrm{g}]}\end{array}$ & $\begin{array}{l}\text { B: } \quad \mathrm{Na} \\
\text { silicate }[\mathrm{g}]\end{array}$ & $\begin{array}{l}\text { C: Metakaolin } \\
\text { [g] }\end{array}$ & & \\
\hline MK-Na-N1 & $-1(6.24)$ & $-1(7.95)$ & $-1(50)$ & 18.1 & 18.2 \\
\hline MK-Na-N2 & $+1(8.67)$ & $-1(7.95)$ & $-1(50)$ & 19.2 & 19.3 \\
\hline MK-Na-N3 & $-1(6.24)$ & $+1(9.72)$ & $-1(50)$ & 18.3 & 18.3 \\
\hline MK-Na-N4 & $+1(8.67)$ & $+1(9.72)$ & $-1(50)$ & 19.7 & 19.7 \\
\hline MK-Na-N5 & $-1(6.24)$ & $-1(7.95)$ & $+1(80)$ & 14.5 & 14.7 \\
\hline MK-Na-N6 & $+1(8.67)$ & $-1(7.95)$ & $+1(80)$ & 16.3 & 16.0 \\
\hline MK-Na-N7 & $-1(6.24)$ & $+1(9.72)$ & $+1(80)$ & 14.7 & 14.7 \\
\hline MK-Na-N8 & $+1(8.67)$ & $+1(9.72)$ & $+1(80)$ & 16.5 & 17.3 \\
\hline MK-Na-N9 & $-1(6.24)$ & $0(8.84)$ & $0(65)$ & 16.5 & 16.3 \\
\hline MK-Na-N10 & $+1(8.67)$ & $0(8.84)$ & $0(65)$ & 17.3 & 17.7 \\
\hline MK-Na-N11 & $0(7.455)$ & $-1(7.95)$ & $0(65)$ & 17.3 & 17.5 \\
\hline MK-Na-N12 & $0(7.455)$ & $+1(9.72)$ & $0(65)$ & 17.7 & 17.4 \\
\hline MK-Na-N13 & $0(7.455)$ & $0(8.84)$ & $-1(50)$ & 19.1 & 18.8 \\
\hline MK-Na-N14 & $0(7.455)$ & $0(8.84)$ & $+1(80)$ & 15.7 & 15.7 \\
\hline MK-Na-N15 & $0(7.455)$ & $0(8.84)$ & $0(65)$ & 16.6 & 17.1 \\
\hline MK-Na-N16 & $0(7.455)$ & $0(8.84)$ & $0(65)$ & 17.4 & 17.5 \\
\hline MK-Na-N17 & $0(7.455)$ & $0(8.84)$ & $0(65)$ & 17.3 & 17.4 \\
\hline
\end{tabular}


Table 6. The results of the $\mathrm{NH}_{4}{ }^{+}$removal tests with $\mathrm{K}$-based MK-GPs.

\begin{tabular}{|c|c|c|c|c|c|}
\hline \multirow[b]{2}{*}{$\begin{array}{l}\text { Experimental } \\
\text { run (sample) }\end{array}$} & \multicolumn{3}{|l|}{ Factor/level } & \multirow{2}{*}{$\begin{array}{l}\text { Observed } \\
\mathrm{NH}_{4}{ }^{+} \\
\text {adsorption } \\
\text { capacity } \\
{[\mathrm{mg} / \mathrm{g}]} \\
\text { experiment } \\
1\end{array}$} & \multirow{2}{*}{$\begin{array}{l}\text { Observed } \\
\mathrm{NH}_{4}^{+} \\
\text {adsorption } \\
\text { capacity } \\
{[\mathrm{mg} / \mathrm{g}]} \\
\text { experiment } \\
2 \\
\end{array}$} \\
\hline & $\mathrm{A}: \mathrm{KOH}[\mathrm{g}]$ & $\begin{array}{l}\text { B: K silicate } \\
{[\mathrm{g}]}\end{array}$ & $\begin{array}{l}\text { C: } \\
\text { Metakaolin } \\
{[\mathrm{g}]}\end{array}$ & & \\
\hline MK-K-N1 & $-1(8.29)$ & $-1(8.07)$ & $-1(50)$ & 13.9 & 13.7 \\
\hline MK-K-N2 & $+1(11.33)$ & $-1(8.07)$ & $-1(50)$ & 14.9 & 14.9 \\
\hline MK-K-N3 & $-1(8.29)$ & $+1(9.86)$ & $-1(50)$ & 13.6 & 13.7 \\
\hline MK-K-N4 & $+1(11.33)$ & $+1(9.86)$ & $-1(50)$ & 15.3 & 15.7 \\
\hline MK-K-N5 & $-1(8.29)$ & $-1(8.07)$ & $+1(80)$ & 10.1 & 10.5 \\
\hline MK-K-N6 & $+1(11.33)$ & $-1(8.07)$ & $+1(80)$ & 11.9 & 12.1 \\
\hline MK-K-N7 & $-1(8.29)$ & $+1(9.86)$ & $+1(80)$ & 10.7 & 10.7 \\
\hline MK-K-N8 & $+1(11.33)$ & $+1(9.86)$ & $+1(80)$ & 13.3 & 12.5 \\
\hline MK-K-N9 & $-1(8.29)$ & $0(8.965)$ & $0(65)$ & 10.9 & 11.3 \\
\hline MK-K-N10 & $+1(11.33)$ & $0(8.965)$ & $0(65)$ & 13.5 & 13.5 \\
\hline MK-K-N11 & $0(9.81)$ & $-1(8.07)$ & $0(65)$ & 12.5 & 12.2 \\
\hline MK-K-N12 & $0(9.81)$ & $+1(9.86)$ & $0(65)$ & 12.9 & $17.5^{a}$ \\
\hline MK-K-N13 & $0(9.81)$ & $0(8.965)$ & $-1(50)$ & 14.4 & $18.2^{\mathrm{a}}$ \\
\hline MK-K-N14 & $0(9.81)$ & $0(8.965)$ & $+1(80)$ & 11.5 & 10.9 \\
\hline MK-K-N15 & $0(9.81)$ & $0(8.965)$ & $0(65)$ & 12.9 & 12.1 \\
\hline MK-K-N16 & $0(9.81)$ & $0(8.965)$ & $0(65)$ & 12.7 & 12.1 \\
\hline MK-K-N17 & $0(9.81)$ & $0(8.965)$ & $0(65)$ & 13.1 & 12.3 \\
\hline
\end{tabular}

$\mathrm{a}=$ Outlier excluded from the experimental data.

Quadratic models were fitted to the experimental data using the multiple linear regression technique (MLR). The significance of the models was tested by means of analysis of variance (ANOVA). The ANOVA results for the response surface quadratic models are summarized in Tables 7 and 8 . As an overview, the goodness of fit $\left(R^{2}\right)$ and goodness of prediction $\left(Q^{2}\right)$ for both models were very good.

The model for Na-based MK-GP is discussed first. The important diagnostic tools, $\mathrm{R}^{2}$ and $\mathrm{Q}^{2}$, exhibited high values: 0.979 and 0.959 , respectively. Hence, the model fitted very well to the experimental data and the model also had a very good predictive power. The model coefficients are presented in Fig. 3. There are some insignificant two-factor interaction terms in the model that could be removed. However, the regression model is statistically significant $(p<0.05)$ and there is no lack of fit $(0.641$, Table 7$)$. The normal probability plot of residuals shows that there are no outliers in the experimental data but that two experiments deviated to some extend (Fig. 4). The deviations were MK-NaN8 experiment 1 and MK-Na-N13 experiment 1 (see Table 6). However, they were not excluded from the experimental data. 


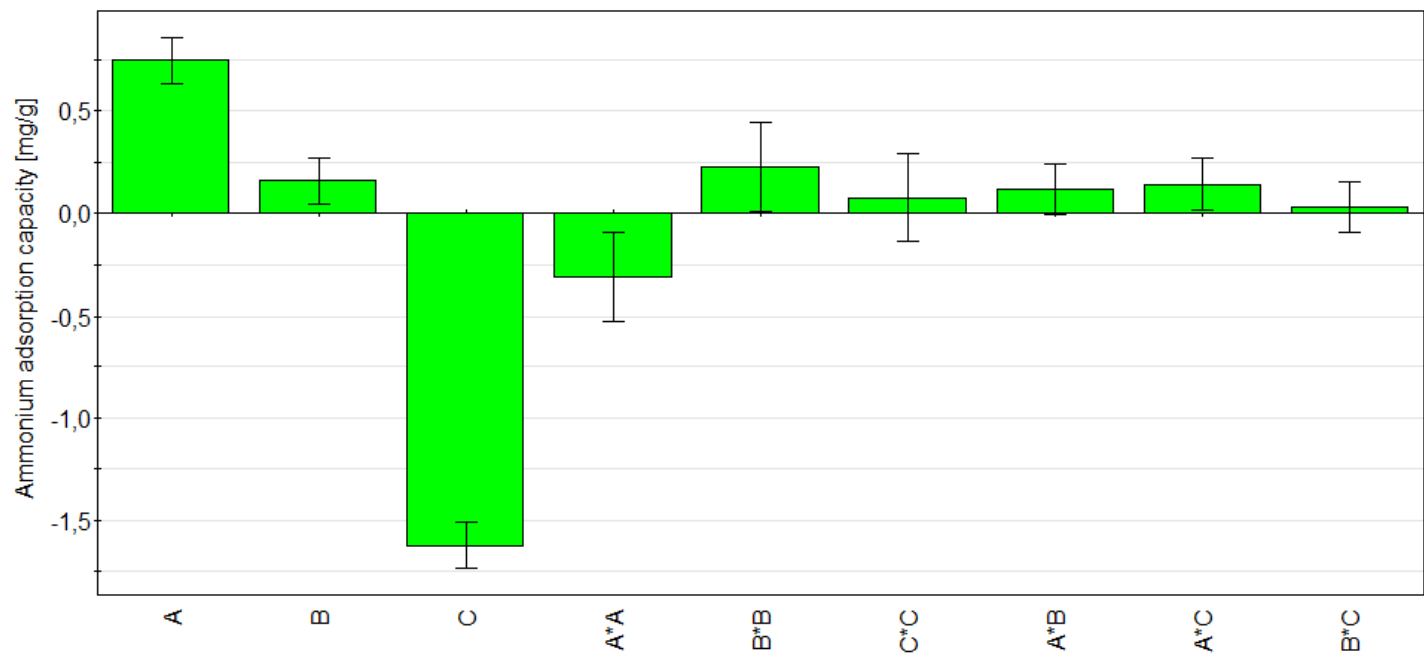

Fig 3. Scaled and centered regression coefficients for the model of the Na-based MKGPs.

Table 7. ANOVA for the response surface quadratic model for Na-based MK-GP. $R^{2}=$ 0.979 and $Q^{2}=0.959$.

\begin{tabular}{llllllll}
\hline $\begin{array}{l}\text { Source } \\
\text { variation }\end{array}$ & $\begin{array}{l}\text { Degree } \\
\text { freedom }\end{array}$ & of & $\begin{array}{l}\text { Sum } \\
\text { squares }\end{array}$ & of & $\begin{array}{l}\text { Mean } \\
\text { square }\end{array}$ & F value & p value \\
\hline Model & 9 & 65.416 & 7.2685 & 123,203 & $\begin{array}{l}7.108 \\
10^{-18}\end{array}$ & $\times$ \\
& & & & & & & \\
Residual & 24 & 1.4159 & 0.058996 & & & \\
Lack of fit & 5 & 0.21620 & 0.043239 & 0.684795 & 0.641 & \\
$\begin{array}{l}\text { Pure error } \\
\text { Total }\end{array}$ & 19 & 1.1997 & 0.063142 & & & \\
corrected & 33 & 66.832 & 2.0252 & & & \\
\hline
\end{tabular}

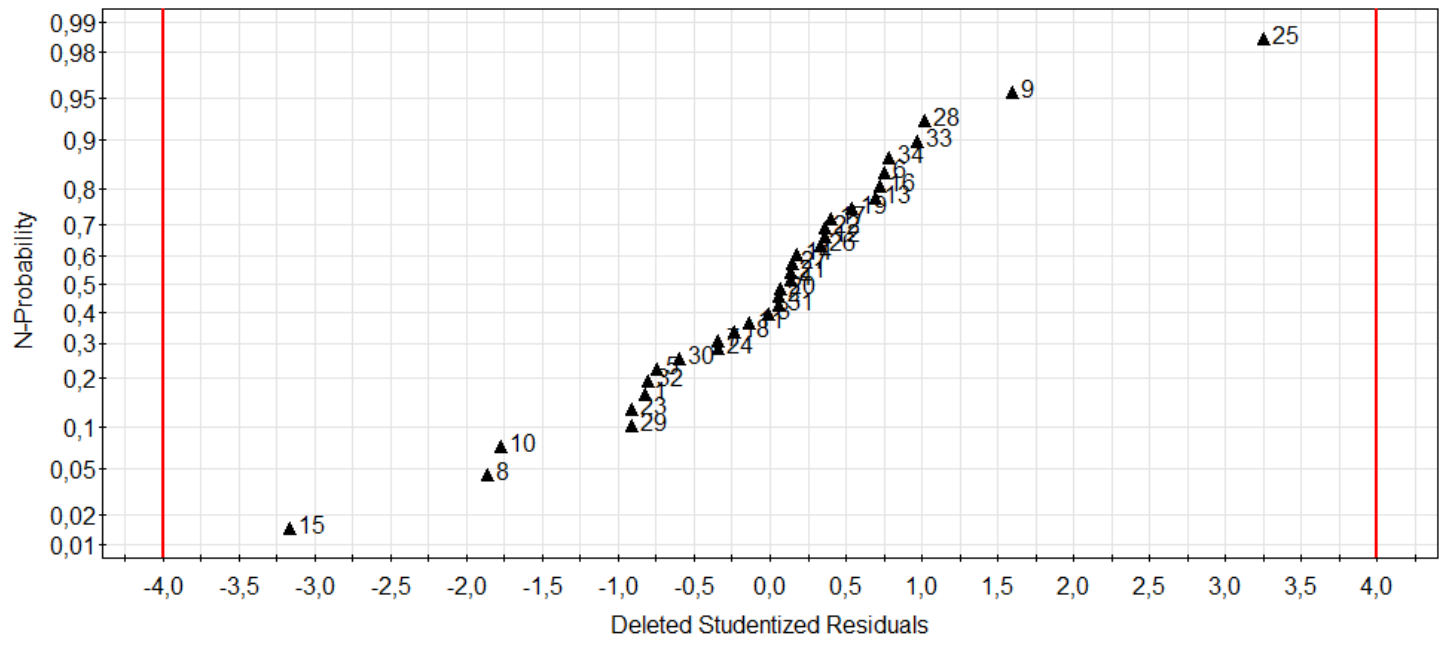

Fig 4. Normal probability plot of residuals for Na-based MK-GPs. 
The obtained regression model for the K-based MK-GPs was also very good $\left(R^{2}=0.964\right.$ and $\left.Q^{2}=0.933\right)$. The model coefficients are presented in Fig. 5 . Again, similarly as with the Na-based series, there are some insignificant two-factor interaction terms in the model that could be removed. However, according to the ANOVA (Table 8), the model was significant and there was no lack of fit. The normal probability plot (Fig. 6) of residuals showed two outliers in the experimental data (shown in Table 6) which were excluded from the data analysis. The observed main effects were similar to that of $\mathrm{Na}$ based MK-GPs.

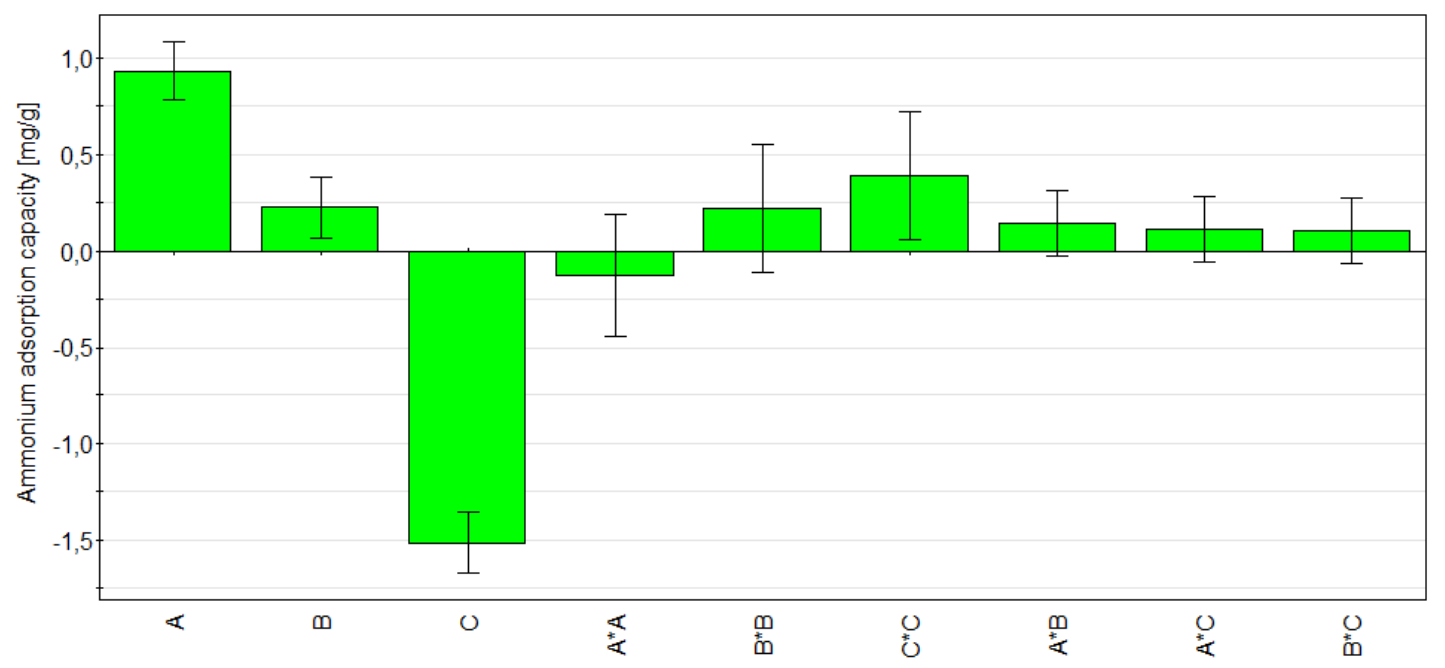

Fig 5. Scaled and centered regression coefficients for the model of the K-based MK-GPs.

Table 8. ANOVA for the response surface quadratic model for K-based MK-GP. $R^{2}=0.964$ and $Q^{2}=0.933$.

\begin{tabular}{|c|c|c|c|c|c|}
\hline $\begin{array}{l}\text { Source of } \\
\text { variation }\end{array}$ & $\begin{array}{l}\text { Degree of } \\
\text { freedom }\end{array}$ & $\begin{array}{l}\text { Sum of } \\
\text { squares }\end{array}$ & $\begin{array}{l}\text { Mean } \\
\text { square }\end{array}$ & F value & $p$ value \\
\hline Model & 9 & 64.187 & 7.1319 & 66.313 & $\begin{array}{l}7.364 \times 10^{-} \\
14\end{array}$ \\
\hline Residual & 22 & 2.3661 & 0.10755 & & \\
\hline Lack of fit & 5 & 0.52727 & 0.10545 & 0.97495 & 0.461 \\
\hline Pure error & 17 & 1.8388 & 0.10816 & & \\
\hline $\begin{array}{l}\text { Total } \\
\text { corrected }\end{array}$ & 32 & 66.553 & 2.1469 & & \\
\hline
\end{tabular}




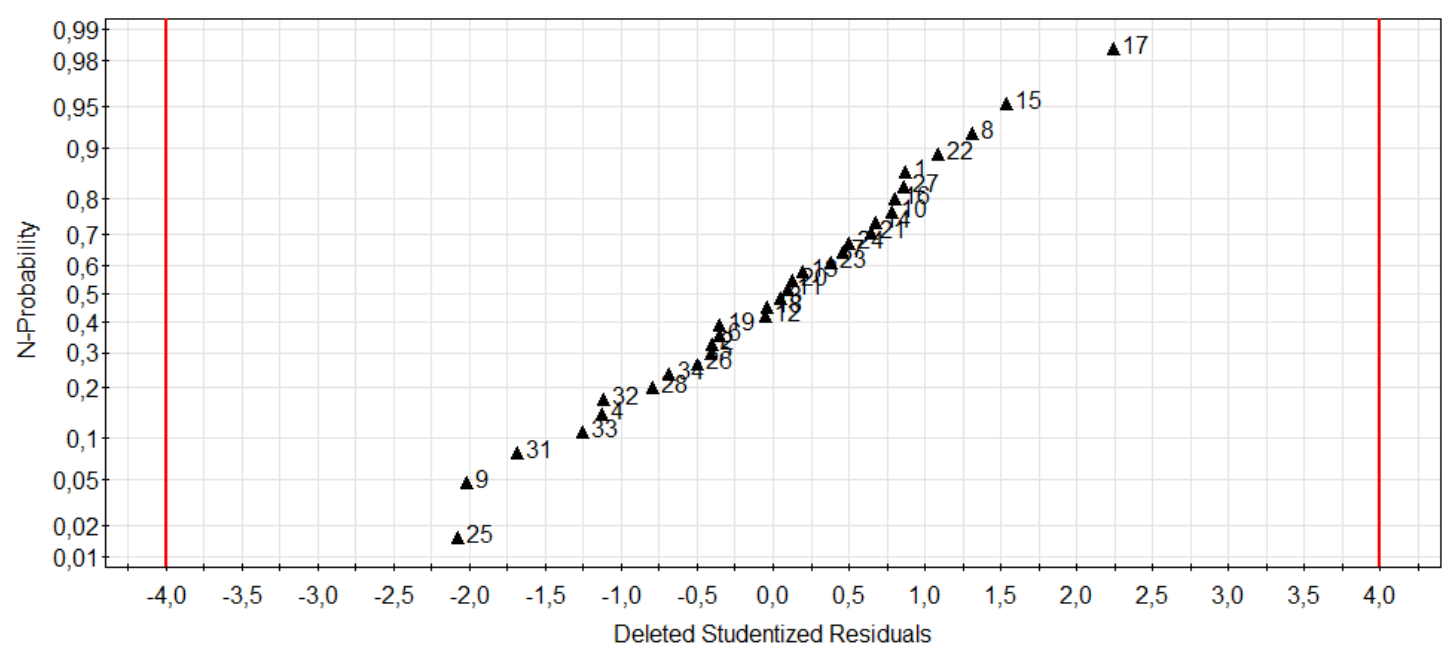

Fig 6. Normal probability plot of K-based MK-GP residuals.

The highest experimental $\mathrm{NH}_{4}{ }^{+}$adsorption capacity $(19.7 \mathrm{mg} / \mathrm{g}$ and $15.5 \mathrm{mg} / \mathrm{g}$ as an average of two repetitions for $\mathrm{Na}$ and $\mathrm{K}$-based series, respectively) was achieved using the highest amount of hydroxide (8.67 or $11.33 \mathrm{~g}$ of $\mathrm{NaOH}$ or $\mathrm{KOH}$, respectively), the highest amount of silicate ( 9.72 or $9.86 \mathrm{~g}$ of $\mathrm{Na}$ or K silicate, respectively) and the lowest amount of metakaolin (50 g) in the MK-GP preparation (Tables 5 and 6). The predicted values by of $\mathrm{NH}_{4}{ }^{+}$adsorption capacity were in good agreement with the experimental results (Fig. 7 and 8). These observed effects are most likely due to the more efficient conversion of metakaolin into geopolymer: metakaolin very low $\mathrm{NH}_{4}{ }^{+}$adsorption capacity [18]. Increased amount of soluble silica and hydroxide increase the degree of geopolymerization through an improved dissolution of precursor alumino-silicate [33, 34]. On the other hand, decreasing the amount metakaolin minimizes the amount of unreacted metakaolin in the final product. As the ammonium removal was observed as adsorption capacity per unit weight $(\mathrm{mg} / \mathrm{g})$, the amount of unreacted metakaolin directly reduces the capacity. Finally, the reason why the $\mathrm{Na}$ based series resulted into higher $\mathrm{NH}_{4}{ }^{+}$removal than the $\mathrm{K}$ based series is not as clear. It has been noted that $\mathrm{Na}^{+}$ results into more ordered framework than $\mathrm{K}^{+}$which indicates that the $\mathrm{Na}$ based series might have more favourable void structure for $\mathrm{NH}_{4}{ }^{+}$adsorption [2]. 


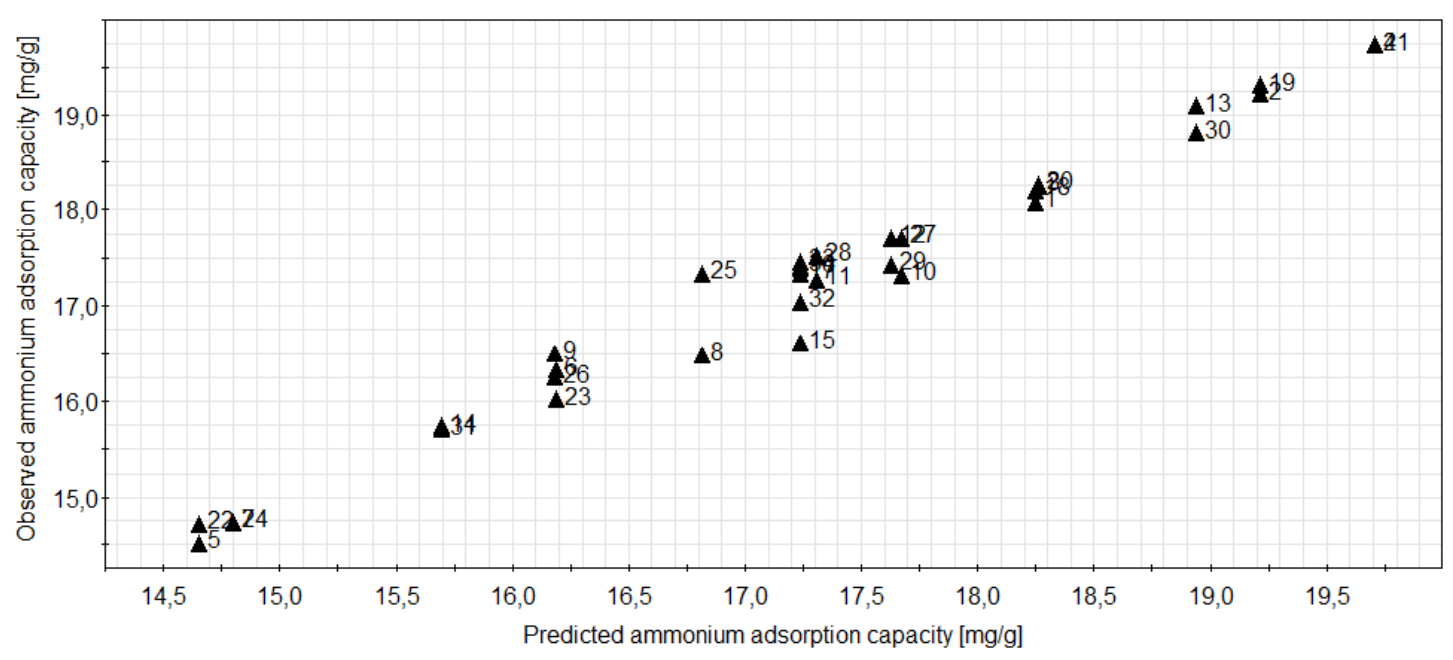

Fig. 7. Observed response vs. model predicted response plot for Na-based MK-GPs.

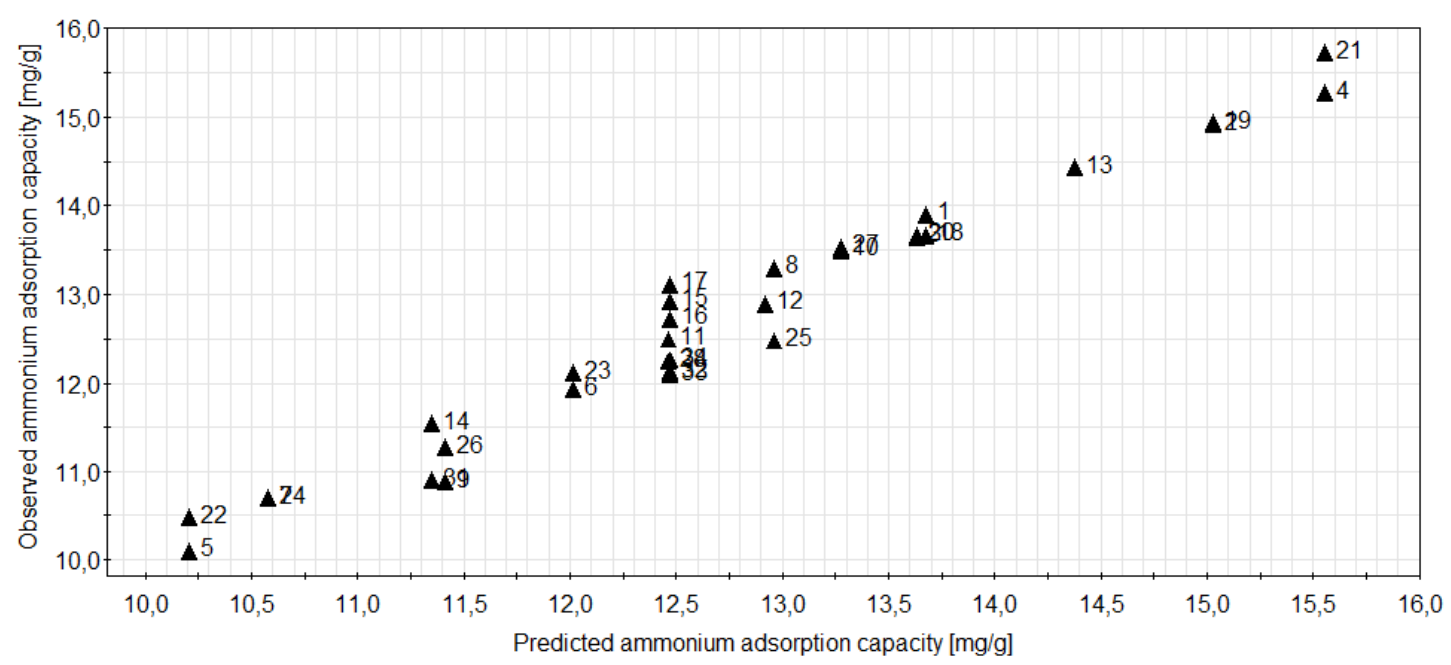

Fig. 8. Observed response vs. model predicted response plot for K-based MK-GPs.

The response surface plots (Figs. 9 and 10) show the effects of the amounts of hydroxide and metakaolin on the $\mathrm{NH}_{4}{ }^{+}$adsorption efficiency. The third factor (the amount of silicate) was fixed at a high level (see Table 1) since it gave the best results in the experiments. According to the fitted models, an even higher $\mathrm{NH}_{4}{ }^{+}$adsorption capacity could be possibly obtained by further increasing the amounts of hydroxide or silicate or by decreasing the amount metakaolin. However, viscosity of the alkaline solution increases if the weight percentage of solids is increased [35]. Additionally, the $\mathrm{SiO}_{2} / \mathrm{M}_{2} \mathrm{O}$ $(\mathrm{M}=\mathrm{Na}$ or $\mathrm{K}$ ) molar ratio of the alkaline solution also affects the viscosity and in fact there is a minimum viscosity area in terms of $\mathrm{SiO}_{2} / \mathrm{M}_{2} \mathrm{O}$ which gets narrower as the percentage of solids increases [35]. The optimum $\mathrm{SiO}_{2} / \mathrm{Na}_{2} \mathrm{O}$ molar ratio of alkaline solution would be approx. 1.8 in terms of viscosity [35]. In the present study, the $\mathrm{SiO}_{2} / \mathrm{Na}_{2} \mathrm{O}$ molar ratio (in the $\mathrm{Na}$-based series) of alkaline solution was $0.27-0.57$ and it was noticed that if the alkaline solutions were allowed to stand several days, crystals were formed especially in those alkaline solutions containing more concentrated 
hydroxide. One advantage of a K-based alkali solution is significantly lower viscosity compared to a Na-based alkali solution at the same concentration [36]. Subsequently, the precipitate formation when using $\mathrm{KOH}$ and $\mathrm{K}$ silicate is not as severe. Due to the aforementioned reasons, the $\mathrm{NaOH}$ or $\mathrm{KOH}$ concentration cannot be increased arbitrarily. The amount of metakaolin, on the other hand, cannot be varied outside the current range or the workability of the geopolymer paste becomes difficult.

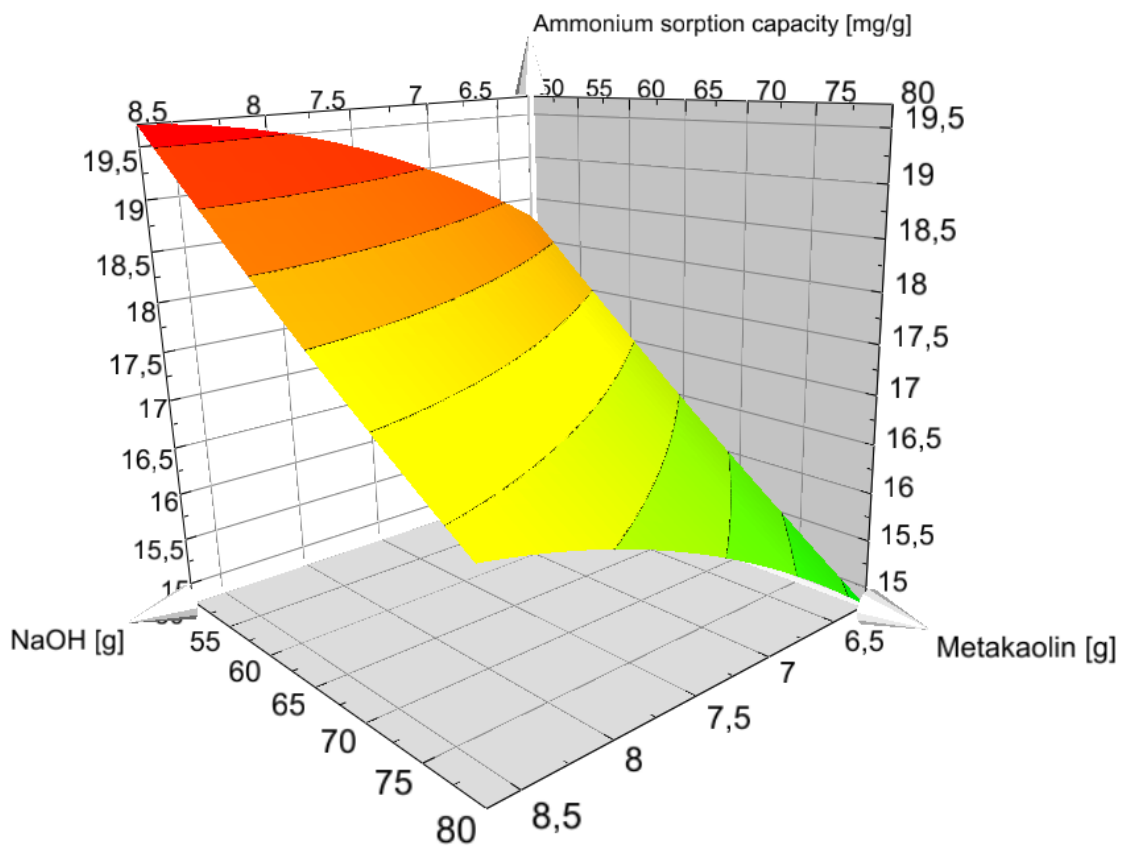

Fig. 9. Response surface plot of the $\mathrm{NaOH}$ and metakaolin amounts (in grams) on $\mathrm{NH}_{4}{ }^{+}$ adsorption capacity at a sodium silicate mass of $9.72 \mathrm{~g}$. 


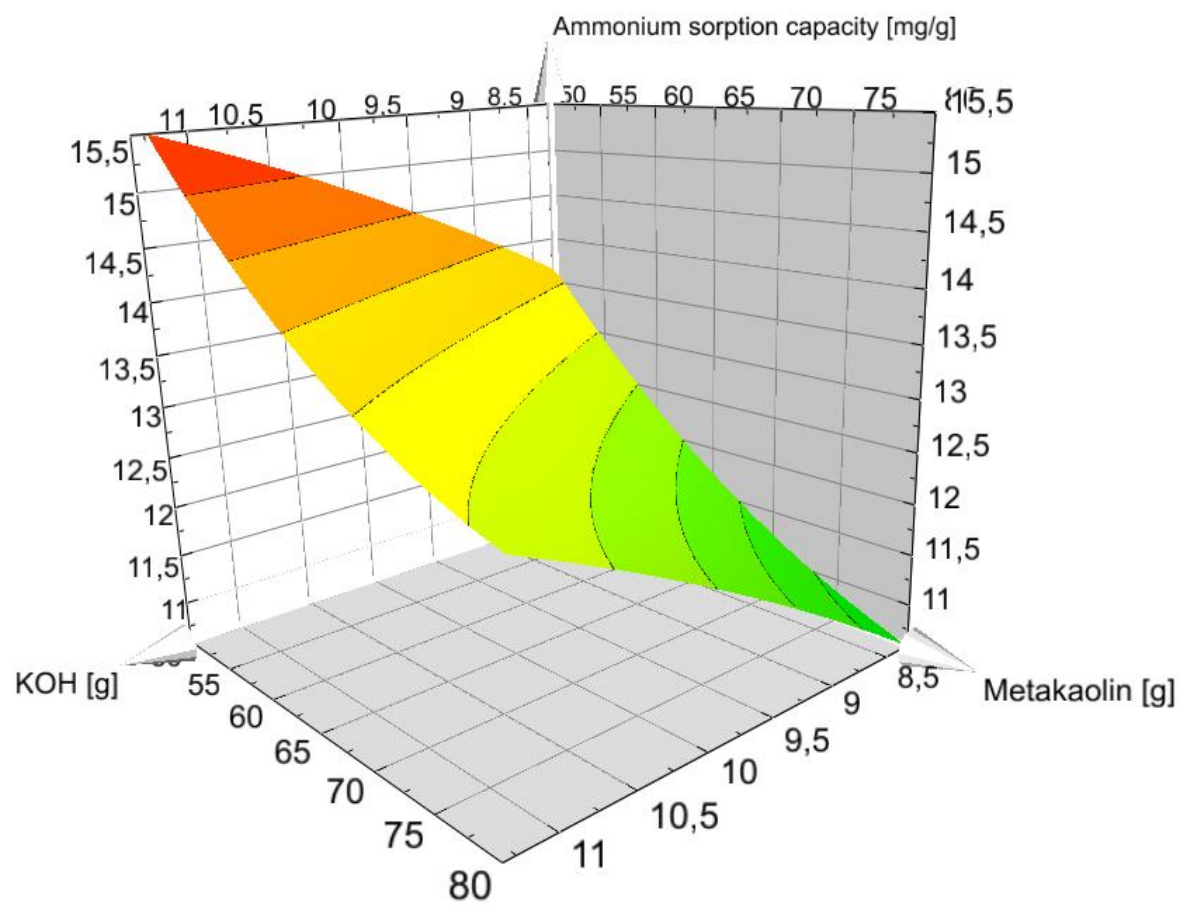

Fig. 10. Response surface plot of the $\mathrm{KOH}$ and metakaolin amounts (in grams) on $\mathrm{NH}_{4}{ }^{+}$ adsorption capacity at a potassium silicate mass of $9.86 \mathrm{~g}$.

\section{Conclusions}

The aim of this study was to find the optimum composition for metakaolin geopolymer in terms of $\mathrm{NH}_{4}{ }^{+}$adsorption capacity. The response surface methodology based on a face centered central composite design was used to evaluate the effects of and to find optimum values for three factors: the amounts of hydroxide, silicate, and metakaolin used for the geopolymer preparation. The $\mathrm{NH}_{4}{ }^{+}$adsorption capacity was used as the response function. Furthermore, the effect of charge balancing cation (i.e. $\mathrm{K}^{+}$or $\mathrm{Na}^{+}$) was studied by conducting all experiments with sodium and potassium hydroxide and silicate.

The following main conclusions can be drawn:

- The highest $\mathrm{NH}_{4}^{+}$removal with metakaolin geopolymer was achieved when maximizing the amounts of $\mathrm{NaOH}$ and $\mathrm{Na}$ silicate and minimizing the amount of metakaolin in the preparation of geopolymer. This was most likely due to the more efficient conversion of metakaolin into geopolymer by adjusting the aforementioned parameters.

- Sodium-based metakaolin geopolymers had $27-48 \%$ higher $\mathrm{NH}_{4}{ }^{+}$adsorption capacity than potassium-based metakaolin geopolymers. There was no conclusive explanation for this observation but it could be related to the void 
structure: $\mathrm{Na}^{+}$has been reported to result a more ordered alumino-silicate framework that $\mathrm{K}^{+}$. All samples were still $\mathrm{X}$-ray amorphous.

- According to the fitted models, the maximum adsorption capacity seemed to be outside the studied range of factors (i.e., an even higher amount of $\mathrm{NaOH}$ and $\mathrm{Na}$ silicate and a lower amount of metakaolin would have been required).

- In practice, the amounts of $\mathrm{NaOH}$ or $\mathrm{Na}$ silicate cannot be further increased due to the precipitation tendency of a highly concentrated alkali solution with the $\mathrm{SiO}_{2} / \mathrm{M}_{2} \mathrm{O}(\mathrm{M}=\mathrm{Na}$ or $\mathrm{K})$ molar ratio much higher or lower than 1.8. The amount of metakaolin cannot be changed outside the studied range or the workability of geopolymer paste becomes poor. According to the literature, the potassium silicate solutions are not as prone to precipitation as sodium silicate solutions.

\section{Acknowledgements}

This work was supported by the Finnish Funding Agency for Innovation (TEKES) [grant number 4096/31/2014, project GeoSorbents]. Authors gratefully acknowledge the contributions of Henrik Romar, Riikka Juhola, Tom Heyninck, Esther Takaluoma, Kai Tiihonen, and Marjukka Hyyryläinen in the laboratory work.

\section{References}

[1] Provis JL, Van Deventer JSJ (2009) Introduction to geopolymers. In: Provis JL, Van Deventer JSJ (eds) Geopolymers - Structure, processing, properties and industrial applications, CRC Press, Boca Raton, pp 1-11

[2] Duxson P, Fernández-Jiménez A, Provis JL, Lukey GC, Palomo A, Van Deventer JSJ (2007) Geopolymer technology: the current state of the art, J Mater Sci 42:2917-2933

[3] Le-ping L, Xue-min C, Shu-heng Q, Jun-li Y, Lin Z (2010) Preparation of phosphoric acid-based porous geopolymers, Appl Clay Sci 50:600-603

[4] Liu Y, Yan C, Zhang Z, Wang H, Zhou S, Zhou W (2016) A comparative study on fly ash, geopolymer and faujasite block for $\mathrm{Pb}$ removal from aqueous solution, Fuel 185:181-189

[5] Duan P, Yan C, Zhou W, Ren D (2016) Development of fly ash and iron ore tailing based porous geopolymer for removal of Cu (II) from wastewater, Ceram Int 42:1350713518.

[6] Mužek MN, Svilovic S, Ugrina M, Zelic J (2015) Removal of copper and cobalt ions by fly ash-based geopolymer from solutions-equilibrium study, Desalin Water Treat 57: 10689-10699.

[7] Ge Y, Cui X, Kong Y, Li Z, He Y, Zhou Q (2015) Porous geopolymeric spheres for removal of $\mathrm{Cu}(\mathrm{II})$ from aqueous solution: Synthesis and evaluation, J Hazard Mater 283:244-251 
[8] Khan MI, Min TK, Azizli K, Sufian S, Ullah H, Man Z (2015) Effective removal of methylene blue from water using phosphoric acid based geopolymers: synthesis, characterizations and adsorption studies, RSC Adv 5:61410-61420

[9] Al-Harahsheh MS, Al Zboon K, Al-Makhadmeh L, Hararah M, Mahasneh M (2015) Fly ash based geopolymer for heavy metal removal: A case study on copper removal, J Environ Chem Eng, 3:1669-1677

[10] Runtti H, Luukkonen T, Niskanen M, Tuomikoski S, Kangas T, Tynjälä P, Tolonen E, Sarkkinen M, Kemppainen K, Rämö J (2016) Sulphate removal over barium-modified blast-furnace-slag geopolymer, J Hazard Mater, 317: 373-384.

[11] Luukkonen $T$, Runtti $H$, Niskanen $M$, Tolonen $E$, Sarkkinen $M$, Kemppainen $K$, Rämö J, Lassi U (2016) Simultaneous removal of $\mathrm{Ni}(\mathrm{II}), \mathrm{As}(\mathrm{III})$, and $\mathrm{Sb}(\mathrm{III})$ from spiked mine effluent with metakaolin and blast-furnace-slag geopolymers, J Environ Manage 166:579-588

[12] Ge Y, Yuan Y, Wang K, He Y, Cui X (2015) Preparation of geopolymer-based inorganic membrane for removing $\mathrm{Ni}^{2+}$ from wastewater, J Hazard Mater 299:711-718

[13] Li C, He Y, Tang Q, Wang K, Cui X (2016) Study of the preparation of CdS on the surface of geopolymer spheres and photocatalyst performance, Mater Chem Phys 178:204-210

[14] Gasca-Tirado JR, Manzano-Ramírez A, Vazquez-Landaverde PA, Herrera-Díaz El, Rodríguez-Ugarte ME, Rubio-Ávalos JC, Amigó-Borrás V, Chávez-Páez M (2014) Ionexchanged geopolymer for photocatalytic degradation of a volatile organic compound, Mater Lett 134:222-224

[15] Alshaaer M, El-Eswed B, Yousef RI, Khalili F, Rahier H (2016) Development of functional geopolymers for water purification, and construction purposes, J Saudi Chem Soc, 20:S85-S92

[16] Hamaideh A, Al-Qarallah B, Hamdi MR, Mallouh SAA, Alshaaer M (2014) Synthesis of Geopolymers Using Local Resources for Construction and Water Purification, J Water Res Protection 6:507-513

[17] Silva I, Castro-Gomes J, Albuquerque A (2012) Mineral Waste Geopolymeric Artificial Aggregates as Alternative Materials for Wastewater-Treatment Processes: Study of Structural Stability and pH Variation in Water, J Mater Civ Eng 24:623-628

[18] Luukkonen T, Sarkkinen M, Kemppainen K, Rämö J, Lassi U (2016) Metakaolin geopolymer characterization and application for ammonium removal from model solutions and landfill leachate, Appl Clay Sci 119, Part 2:266-276

[19] Jha B, Singh D (2011) A review on synthesis, characterization and industrial applications of flyash zeolites, J Mat Education 33:65-132 
[20] Wang S, Li L, Zhu ZH (2007) Solid-state conversion of fly ash to effective adsorbents for Cu removal from wastewater, J Hazard Mater 139:254-259

[21] Li L, Wang S, Zhu Z (2006) Geopolymeric adsorbents from fly ash for dye removal from aqueous solution, J Colloid Interface Sci 300:52-59

[22] Tang Q, He Y, Wang Y, Wang K, Cui X (2016) Study on synthesis and characterization of ZSM-20 zeolites from metakaolin-based geopolymers, Appl Clay Sci 129:102-107

[23] Lee N, Khalid HR, Lee H (2016) Synthesis of mesoporous geopolymers containing zeolite phases by a hydrothermal treatment, Microporous Mesoporous Mater 229:2230

[24] Criado M, Fernández-Jiménez A, Palomo A (2007) Alkali activation of fly ash: effect of the $\mathrm{SiO}_{2} / \mathrm{Na}_{2} \mathrm{O}$ ratio: Part I: FTIR study, Microporous Mesoporous Mater 106:180-191

[25] Lundstedt T, Seifert E, Abramo L, Thelin B, Nyström Å, Pettersen J, Bergman R (1998) Experimental design and optimization, Chemometrics Intellig Lab Syst 42:3-40

[26] Provis JL, Lukey GC, Van Deventer JSJ (2005) Do geopolymers actually contain nanocrystalline zeolites? a reexamination of existing results, Chem Mater 17:3075-3085

[27] Barbosa VFF, MacKenzie KJD, Thaumaturgo C (2000) Synthesis and characterisation of materials based on inorganic polymers of alumina and silica: Sodium polysialate polymers, J Inord Mater 2:309-317

[28] López FJ, Sugita S, Tagaya M, Kobayashi T (2014) Metakaolin-Based Geopolymers for Targeted Adsorbents to Heavy Metal Ion Separation, J Mater Sci Chem Eng 2:16-27

[29] Duan J, Li J, Lu Z (2015) One-step facile synthesis of bulk zeolite A through metakaolin-based geopolymer gels, J Porous Mater, 22:1519-1526

[30] Khale D, Chaudhary R (2007) Mechanism of geopolymerization and factors influencing its development: a review, J Mater Sci, 42:729-746

[31] Marcus Y (1988) Ionic radii in aqueous solutions, Chem Rev 88:1475-1498

[32] Luukkonen T, Věžníková K, Tolonen E, Runtti H, Yliniemi J, Hu T, Kemppainen K, Lassi $U$ (2017) Removal of ammonium from municipal wastewater with powdered and granulated metakaolin geopolymer, Environ Technol. doi: 10.1080/09593330.2017.1301572

[33] Bignozzi MC, Manzi S, Natali ME, Rickard WDA, Van Riessen A (2014) Room temperature alkali activation of fly ash: The effect of $\mathrm{Na}_{2} \mathrm{O} / \mathrm{SiO}_{2}$ ratio, Constr Build Mater 69:262-270

[34] Rattanasak U, Chindaprasirt P (2009) Influence of $\mathrm{NaOH}$ solution on the synthesis of fly ash geopolymer, Minerals Eng 22:1073-1078 
[35] Weldes HH, Lange KR (1969) Properties of soluble silicates 61:29-44

[36] Davidovits J (2011) Geopolymer chemistry \& applications 3rd edn. Institut Geopolymere, Saint-Quentin 